\title{
Correction to: Tetraspanin-enriched microdomains regulate digitation junctions
}

\author{
Chao Huang ${ }^{1}$. Chenying Fu ${ }^{1}$. Jonathan D. Wren ${ }^{2}$ Xuejun Wang ${ }^{1}$. Feng Zhang ${ }^{1}$. Yanhui H. Zhang ${ }^{3}$. \\ Samuel A. Connell ${ }^{4} \cdot$ Taosheng Chen $^{4} \cdot$ Xin A. Zhang ${ }^{1}$
}

Published online: 8 September 2018

(c) Springer Nature Switzerland AG 2018

\section{Correction to: \\ Cellular and Molecular Life Sciences (2018) 75:3423-3439 \\ https://doi.org/10.1007/s00018-018-2803-2}

In the original publication, one of the co-author's name, abstract text and the legend to Table 1 were incorrectly published. The correct name for the seventh author should read as 'Samuel A. Connell'.

In abstract section, the text currently reads "Tetraspanins co-emerged with multi-cellular organisms during evolution are typically localized at the cell-cell interface, and form tetraspanin-enriched microdomains (TEMs) by associating with each other and other membrane molecules." The correct text should read as "Tetraspanins co-emerged with multi-cellular organisms during evolution and are typically localized at the cell-cell interface".

Table 1 legend currently read as 'Genes predicted to be associated with microvilli by GAMMA' the correct legend should read as 'TEM genes predicted to be associated with mirovilli by GAMMA'.

The original article can be found online at https://doi.org/10.1007/ s00018-018-2803-2.

Xin A. Zhang

xin-zhang-1@ouhsc.edu

1 Stephenson Cancer Center and Department of Physiology, University of Oklahoma Health Sciences Center, BRC Building West Room 1474, 975 N.E. 10th Street, Oklahoma City, OK 73104, USA

2 Oklahoma Medical Research Foundation, Oklahoma City, OK, USA

3 University of Tennessee Health Science Center, Memphis, TN, USA

4 St. Jude Children's Research Hospital, Memphis, TN, USA 